\title{
Garder la bonne direction - Quelques résultats de l'Initiative sur l'évaluation de l'amélioration des services (IEAS-SEEI)

\author{
Lindsey George et Janet Durbin
}

\begin{abstract}
Voici un numéro spécial de Canadian Journal of Community Mental Health/Revue canadienne de santé mentale communautaire dont nous sommes très heureux d'avoir été les directeurs scientifiques invités; à ce titre, nous avons eu le privilège de contribuer à l'immense travail de plusieurs chercheurs qui souhaitaient participer au développement des connaissances dans le domaine de la santé mentale communautaire. Nous sommes reconnaissants à la professeure Paula Goering du rôle déterminant qu'elle a joué pour offrir à des chercheurs et à des intervenants du système de santé mental en Ontario cette occasion unique.

Depuis 2005, le ministère de la Santé et des Soins de longue durée de l'Ontario a accru de $52 \%$ le financement du secteur de la santé mentale communautaire. Ainsi, 167 millions de dollars ont été injectés dans les services de santé mentale communautaire de la province, ce qui s'est traduit par des fonds supplémentaires pour les services de crise, la gestion de cas graves, les solutions de rechange à la judiciarisation et le soutien juridique, le traitement communautaire actif et l'intervention précoce auprès de patients souffrant de psychose. La ministre a aussi engagé des sommes qui ont permis d'évaluer les impacts de ces investissements supplémentaires, et ce sont les résultats de cette évaluation que vous lirez dans ces pages. Les chercheurs qui y ont contribué ont tous tenté, chacun à sa façon, d'établir ce qui fonctionne bien dans le système, et ce que nous pourrions y améliorer : c'était l'objectif de l'Initiative sur l'évaluation de l'amélioration des services (IEAS-SEEI).

C'est l'Unité de recherche et de consultation du Centre for Addiction and Mental Health de Toronto, que dirige la professeure Goering, qui était en charge de l'IEAS. Les projets mis sur pied dans ce cadre ont rassemblé des chercheurs et des intervenants afin de faire avancer la recherche sur l'évaluation du système, afin que nous puissions mieux comprendre les impacts des nouvelles sources de financement en santé mentale communautaire. Après une évaluation par les pairs des articles soumis, à laquelle a contribué la Fondation ontarienne de la santé mentale, neuf études ont été choisies ; elles couvrent un vaste éventail de sujets touchant l'évaluation du système dans son entier ou de certaines de ses composantes, et ont été réalisées grâce
\end{abstract}


à diverses méthodes de recherche. Elles illustrent en détail la complexité de la conception et de la mise en place de services de santé mentale.

Le plus passionnant pour nous dans ce projet a été l'occasion unique qui nous était offerte de relever le défi d'entreprendre un travail d'évaluation complexe - aussi complexe que la réalité que vivent les personnes qui travaillent au quotidien en santé mentale. Notre objectif n'était pas de concevoir des projets de recherche « parfaits » : nous voulions plutôt identifier, avec les personnes ayant des problèmes de santé mentale, leurs proches, les prestataires de soins, les décideurs et les chercheurs, des moyens de mieux comprendre les points forts et les faiblesses du système. Les chercheurs qui signent les articles de ce numéro travaillent dans différents domaines; ils nous ont aidés à concevoir les questions et les méthodes de recherche, à interpréter les données recueillies, et à faire des recommandations sur la base des résultats obtenus. Ce travail est le fruit de la collaboration de plusieurs personnes - et pas seulement des signataires des articles. Nous tenons donc à remercier tous ceux et celles qui y ont participé. Ce type de recherche n'est pas simple à réaliser, mais mieux comprendre ce à quoi nous travaillons est extrêmement stimulant.

Les recherches en évaluation de programmes, par nature, présentent un haut degré de complexité : elles exigent que l'on tienne compte simultanément de nombreux éléments interreliés, ce qui est très difficile - et même parfois impossible. Il est pourtant crucial, pour aller de l'avant, de comprendre comment nous pouvons améliorer les modèles et les systèmes de soins.

\section{Certains défis qui se sont posés}

- Nous avons dû travailler avec des données qui sont imparfaites.

- Impliquer d'autres intervenants exige de l'énergie et du temps.

- Même si les ressources financières ne manquent pas, ce n'est pas nécessairement le meilleur moment de faire une évaluation du travail.

- La mesure de l'impact des nouvelles ressources financières peut parfois n'être réalisée que dans plusieurs années, considérant que la mise en place de nouveaux ou de meilleurs programmes exige beaucoup de temps.

\section{Ce que les études nous ont appris}

- Le nouveau financement en santé mentale communautaire a eu un impact sur ceux et celles qui ont besoin de services en santé mentale.

- Les gens qui ont recours à ces services (et leurs proches) sont, tout bien considérer, satisfaits des services offerts.

- Les systèmes de soins sont complexes. L'injection de nouveaux fonds peut avoir des effets imprévus. Augmenter les ressources peut faire croître les attentes, ce qui peut ainsi entraîner une plus grande demande de services. L'objectif peut donc être d'offrir un meilleur accès au système de soins.

- Mesurer efficacement les impacts du nouveau financement nécessite des données très complètes sur plusieurs domaines relevant de diverses compétences. Les services offerts aux personnes ayant des problèmes de santé mentale sont la responsabilité de différents organismes gouvernementaux et non 
gouvernementaux. Pour mesurer l'impact d'un programme donné, nous devons donc tenir compte de ce qui se fait dans d'autres secteurs - ce qui, en fait, dépasse souvent nos capacités.

- Les données doivent porter sur les individus si l'on veut pouvoir comparer diverses informations recueillies à différentes périodes et dans différents secteurs de services - et même sur des personnes qui ont cessé d'avoir recours au système de santé mentale -, afin d'évaluer de façon précise l'impact du financement en santé mentale communautaire.

- Faire de la recherche sur un domaine extrêmement complexe est stimulant et gratifiant. Cela nous fait prendre conscience de la responsabilité que nous avons envers notre communauté, et nous permet de voir le système de santé mentale de l'intérieur dans toute sa complexité et avec toutes ses imperfections.

Les 11 articles de ce numéro présentent de nombreux résultats obtenus grâce aux recherches qui ont été rendues possibles par l'IEAS. Le premier article analyse le processus qui permet d'obtenir la participation de différents intervenants à une recherche, ce qui est nécessaire pour aboutir à des résultats valides et pertinents. Les articles qui suivent présentent des évaluations des impacts du nouveau financement soit au niveau de l'ensemble de la province soit au niveau de certaines communautés. Enfin, les derniers articles évaluent les expériences de certains organismes. Notons qu'une étude, réalisée dans le cadre du projet Waterloo-Wellington Crisis System Evaluation, n'est pas présentée ici ; elle est toutefois disponible à www.ehealthontario.ca. Dans la communauté de Waterloo-Wellington, le nouveau financement a servi à élargir les services de crise et à améliorer la coordination du système de services de crise, en partie grâce à la création d'un poste de coordonnateur du système ; l'étude décrit certains résultats très positifs, ainsi que les difficultés qu'il reste à régler.

Nous espérons que d'autres efforts seront faits en Ontario pour améliorer les soins offerts aux personnes ayant des problèmes de santé mentale, et pour évaluer les progrès grâce à des recherches collaboratives réalisées par des personnes dont l'objectif est de rendre nos systèmes de soins toujours meilleurs. 
